\title{
ПРОГРАММНОЕ ОБЕСПЕЧЕНИЕ ОЦЕНКИ НЕОПРЕДЕЛЕННОСТИ МИКРОБИОЛОГИЧЕСКОГО АНАЛИЗА ПИЩЕВЫХ ПРОДУКТОВ И КОРМОВ ДЛЯ ЖИВОТНЫХ В СРЕДЕ LabVIEW
}

\author{
Нодари Абелашвили, профессор Грузинского технического университета, Тбилиси, Грузия \\ ORCID ID: https://orcid.org/0000-0003-4002-9177
}

\section{DOI: https://doi.org/10.31435/rsglobal_conf/30052021/7573}

Abstract. In microbiological research, it is difficult to quantify the portion of each step of the analytical process with sufficient accuracy, as the object subject to analysis is a living organism with many species, strains or groups of microorganisms involved.

This paper discusses the issue of assessing and visualizing the uncertainty of the data of quantitative analysis when processing the results of microbiological analysis of food and animal feed.

Results of quantitative analysis are processed in LabVIEW Environment by the software specifically designed for this purpose.

Keywords: standard deviation, standard uncertainty, LabVIEW Programing Environment, microbiological analysis.

Введение. Международный стандарт ISO/TS 19036:2019 "Microbiology of the food chain - Estimation of measurement uncertainty for quantitative determinations" описывает широко распространённый подход в оценке неопределенности измерения для количественного определения веществ в микробиологии пищевой цепи.

Рекомендации по «GUM:1993, Guide to the expression of uncertainty in measurement» [1] описывает широко распространенный стандартный подход, осуществлять оценку неопределенности измерения по отдельности для каждого из источников варьирования, вносящих заметный вклад в общую неопределенность результата измерительного процесса. Суммарная неопределенность в этом случае находится путем использования формальных "принципов распространения неопределенности".

Основная часть. Программное обеспечение оценки неопределённости типа А и типа В результатов измерений создано разработанной компаний „National Instrument” в программной среде LabVIEW (Laboratory Virtual Instrument Engineering Workbench), что дает возможность создать прикладной программный код для моделированных виртуальных и аппаратурных реальных систем измерения, управления, сбора данных и обработки полученных результатов.

Алгоритм для создания кода обработки результатов полученных данных разработан согласно стандарта ISO/TS 19036:2019 а в основу методики реализации обработки данных и вычисления неопределённостей типа $\mathrm{A}$, типа $\mathrm{B}$, суммарной стандартной и расширенной неопределённости соответствует рекомендациям по «GUM:1993, Guide to the expression of uncertainty in measurement». [2,4].

Созданий графический код в среде LabVIEW представляет собой единство расположенных слоями информационных окон (рис. 1), так что на каждом из них выполняются расчеты определенного характера, среди них:

- «Стандартное отклонение»;

- «Наблюдатель А», «Наблюдатель В»;

- «Прибор 1», «Прибор 2»;

- «Протокол испытаний»;

Первый слой программного обеспечения, показаний на рис. 1, уделяется вычислению неопределенности типа А, или что для данного случая, тоже самое, что стандартное отклонение воспроизводимости $S_{R}$, как результат полученного в одной серии двух наблюдателей с помощью статистических методов вычислений. 
Согласно стандарту ISO/TS 19036:2006/2014 стандартное отклонение воспроизводимости $S_{R}$ микробиологического эксперимента вычисляется по формуле: [3].

$$
S_{R}=\sqrt{\frac{1}{n} \sum_{i=1}^{n} \frac{\left(y_{i A}-y_{j B}\right)^{2}}{2}}
$$

Со своей стороны $y_{i A}=\log _{10}\left(X_{i A}\right)$;

$$
y_{j B}=\log _{10}\left(X_{j B}\right) \text {. }
$$

Где $X_{i A}$ и $X_{j B}$ данные эксперимента наблюдателя 1 и наблюдателя 2.

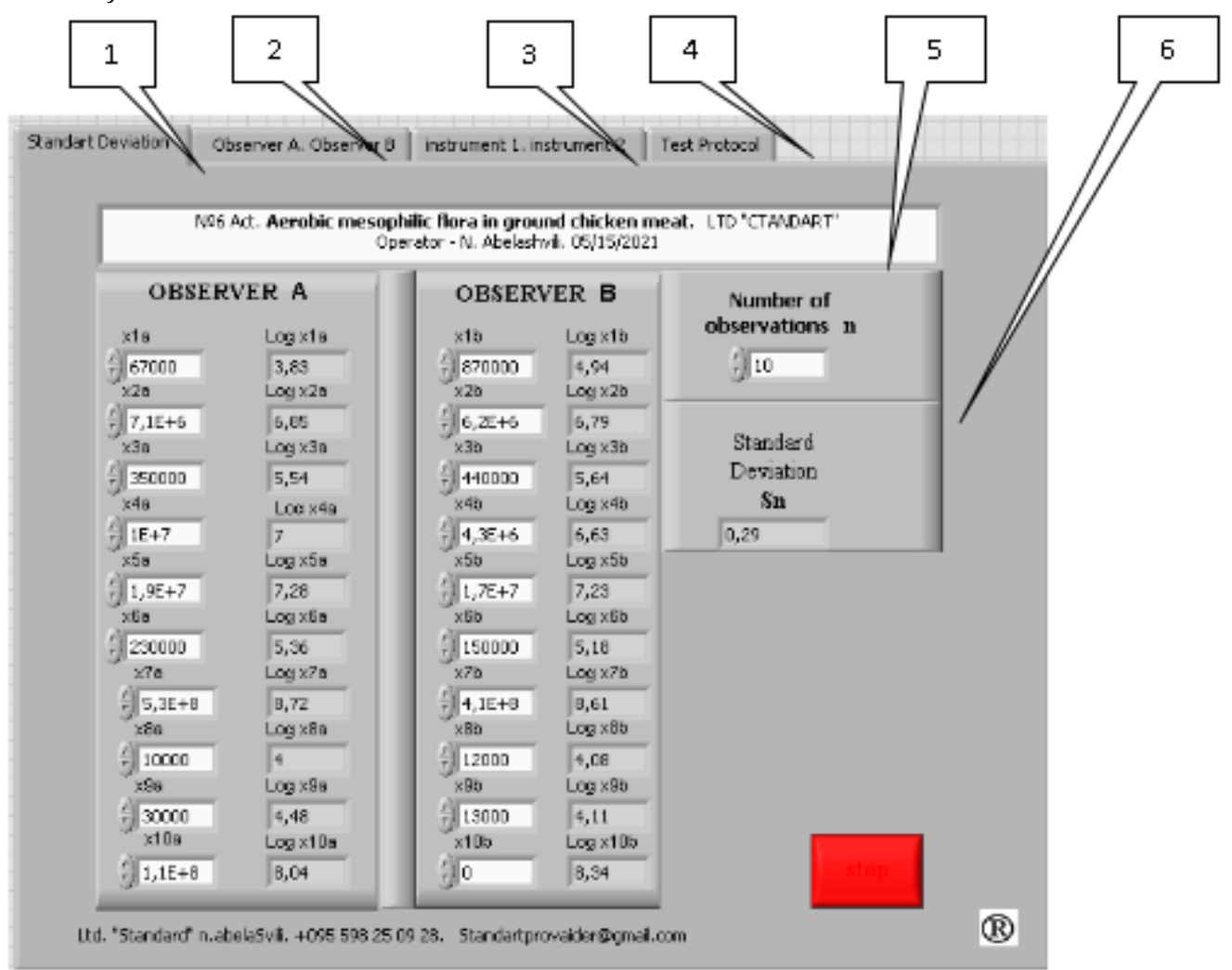

Рис. 1. Окно «Стандартное отклонение» программного обеспечения вычисления неопределённости типа А (Стандартное отклонение $S_{n}$ )

В зоне ввода данных возможно внесение от 3-х до 10-ты разных значений наблюдений. Ввод результатов осуществляется специально выделенных для этого полях $X_{1 A}-X_{10 A}$ и $X_{1 B}-$ $X_{10 в}$ для наблюдателя А и для наблюдателя В.

При вводе значений наблюдений x1a-x10a и x1b-x10b программа вычисляет его логарифм и выдает эти значения для дальнейшего пользования в $\log _{10}\left(\mathrm{X}_{12}\right)-\log _{10}\left(\mathrm{X}_{\mathrm{ara}}\right),\left(\mathrm{X}_{12}\right)$ $\log _{10}\left(\mathrm{X}_{12}\right)$ ячейках.

В этом же окне заполняется, соответствующими записями, поле текстового ввода «полоса данных акта испытаний» (рис. 1, позиция 5). Обычно сюда записывают данные о названии эксперимента, номер и дата акта испытаний, сведения об операторе, условия проведения эксперимента и другие сведения по условию эксперимента.

В соответствующем поле ввода цифровых данных указывается число количества наблюдений (рис. 1, позиция 6).

После того как на первой странице будут заполнены все необходимые поля, переходим на следующий слой «Наблюдатель А», «Наблюдатель В» (рис. 2). На этом слое фактически происходит вычисление неопределённости типа А результатов микробиологического эксперимента наблюдателя А и наблюдателя В. 


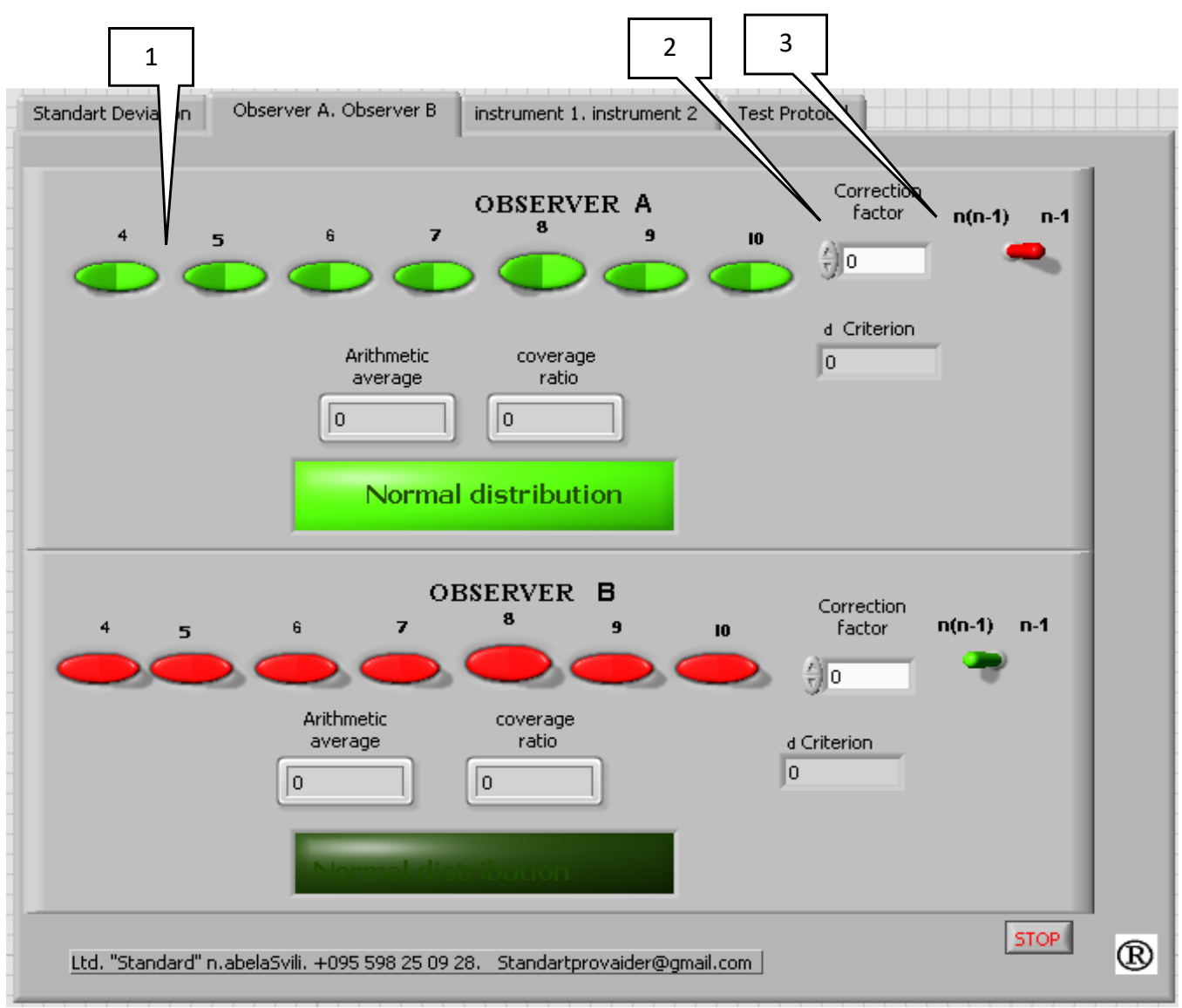

Рис. 2. Окно программного обеспечения «Наблюдатель А», «Наблюдатель В».

Нажатием правой кнопки мыши на клавиши 4-10 красного цвета (рис. 2, позиция 1), они активизируются и меняют красный цвет на зеленый. Процесс активации данных подразумевает определение количества числа наблюдений, для которых вычисляется неопределённость типа А.

Программное обеспечение позволяет вносить, при необходимости, обоснованное значение коэффициента коррекции. По умолчанию установлено значение 1 (рис. 2, позиция 2).

Переключатель (рис. 2. позиция 3) в правом положении на отметке “n-1" для случая, когда данные принадлежат одной серии испытаний. Если данные нескольких серий испытаний, то переключатель в положении $\mathrm{n}(\mathrm{n}-1)$.

Вышеуказанные настройки выполняются как для позиций наблюдателя А, так и для наблюдателя В.

Точностные характеристики, участвующих в эксперименте измерительных средств, могут быть даны по-разному. Программа обеспечивает приведение этих данных на общий вид к предусмотренному алгоритмом вычисления стандартной неопределённости измерения.

Точностные характеристики данные по форме:

- Стандартной неопределённости. В таком случае соответствующие данные заносятся в поле $u_{x}$ (рис. 3 , позиция 1) в тоже время переключатель (рис.3, позиция 3 ) в левом положении «дано» (“Is given”). во всех других случаях переключатель в положении «не дано» (“Not given") (рис. позиция 4); 


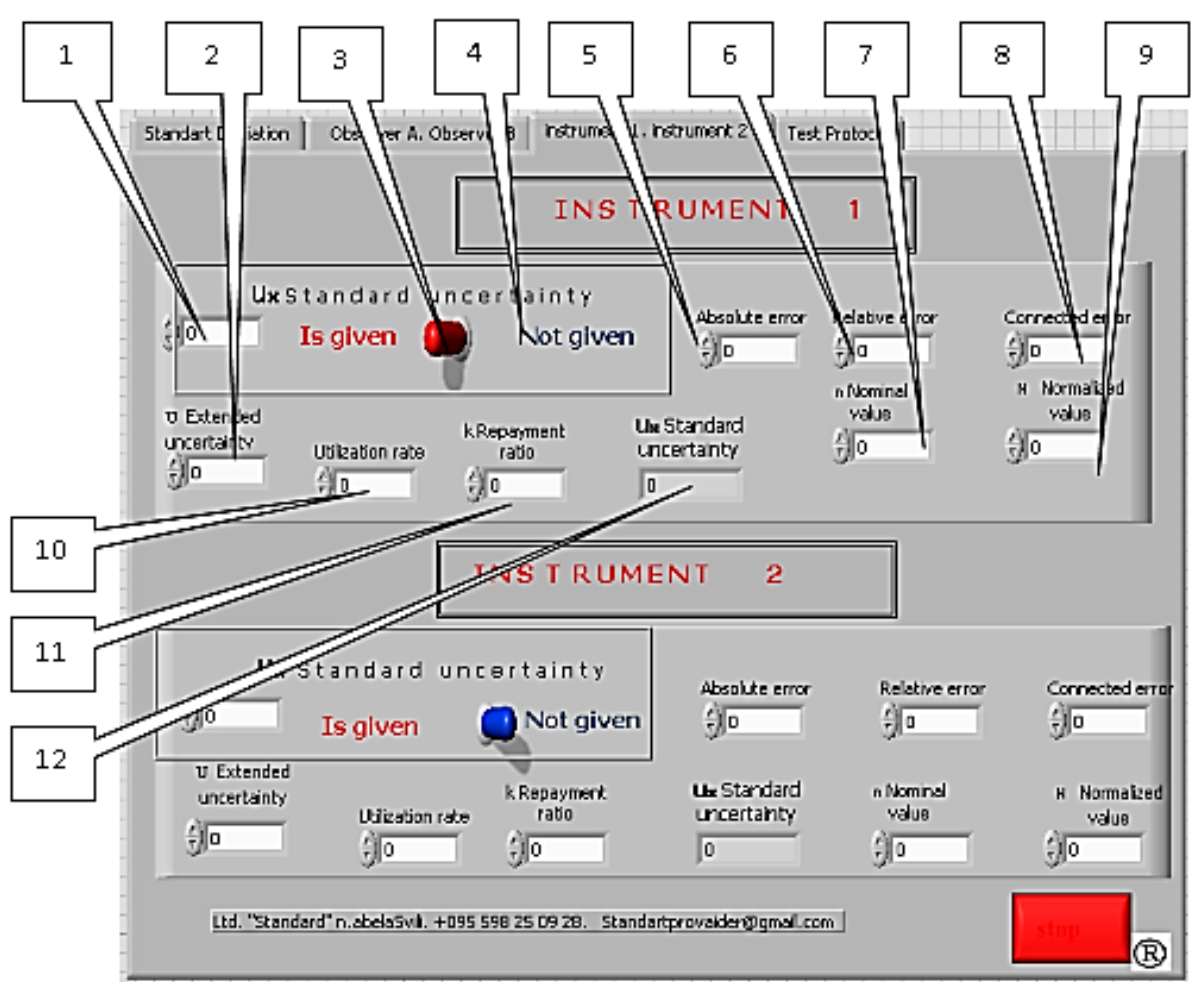

Puc. 3. Окно программного обеспечения "INSTRUMENT 1, INSTRUMENT 2"

- В форме расширенной неопределённости. Данные заносятся в соответствующем поле (рис. 3. позиция 2);

- В виде абсолютной погрешности. Соответствующие данные заносим в поле ввода «абсолютная погрешности» (рис. 3 позиция 5);

- В форме относительной погрешности. Соответствующее значение вводим в поле ввода «Относительная погрешность» (рис. 3, позиция 6). Здесь же в поле ввода «n номинальное значение» вводим измеренное значение данным прибором физической величины» (рис. 3, позиция 7);

- В форме приведенной погрешности. Соответствующее значение водим в поле ввода «Приведенная погрешность» (рис. 3, позиция 8). Здесь же в поле ввода «N нормированное значение» вводим значение «класса точности» из нормативной документации на данное измерительное средство. (рис. 3, позиция 9).

Для всех форм точностных характеристик должно указываться значение т.н. «Коэффициент применяемости», который подразумевает количество применения конкретного измерительного средства в ходе проведения данного эксперимента. (рис. 3 , позиция 10).

Значение «Коэффициента охвата $-k$ » на данное измерительное средство берется из сертификата калибровки или из справочных данных. (рис. 3 , позиция 11 ).

Окончательный результат вычисления стандартной неопределённости для данного измерительного средства показывается в поле индикации «Стандартная неопределённость» (рис. 3, позиция 12).

Описанные манипуляции, при необходимости, надо выполнить и для других измерительных средств.

Результаты исследования. Последний слой программного обеспечения “протокол испытаний”, предназначен для фиксации, сохранения и распечатки результатов эксперимента (рис.4).

В протоколе указывается вся необходимая информация проведения эксперимента (рис. 4 позиции 1, 2, 3. в том числе информация внесённая оператором на первом слое (рис. 1, позиция 5), а также данные эксперимента «Наблюдателя А» и «Наблюдателя В».

В протоколе отражаются также промежуточные результаты (рис. 4 , позиция 6,7 ) и отдельно выделен окончательный результат эксперимента (рис. 4, позиция 8) с указанием расширенной неопределённости и сведений о доверительности результата и значение коэффициента охвата. 


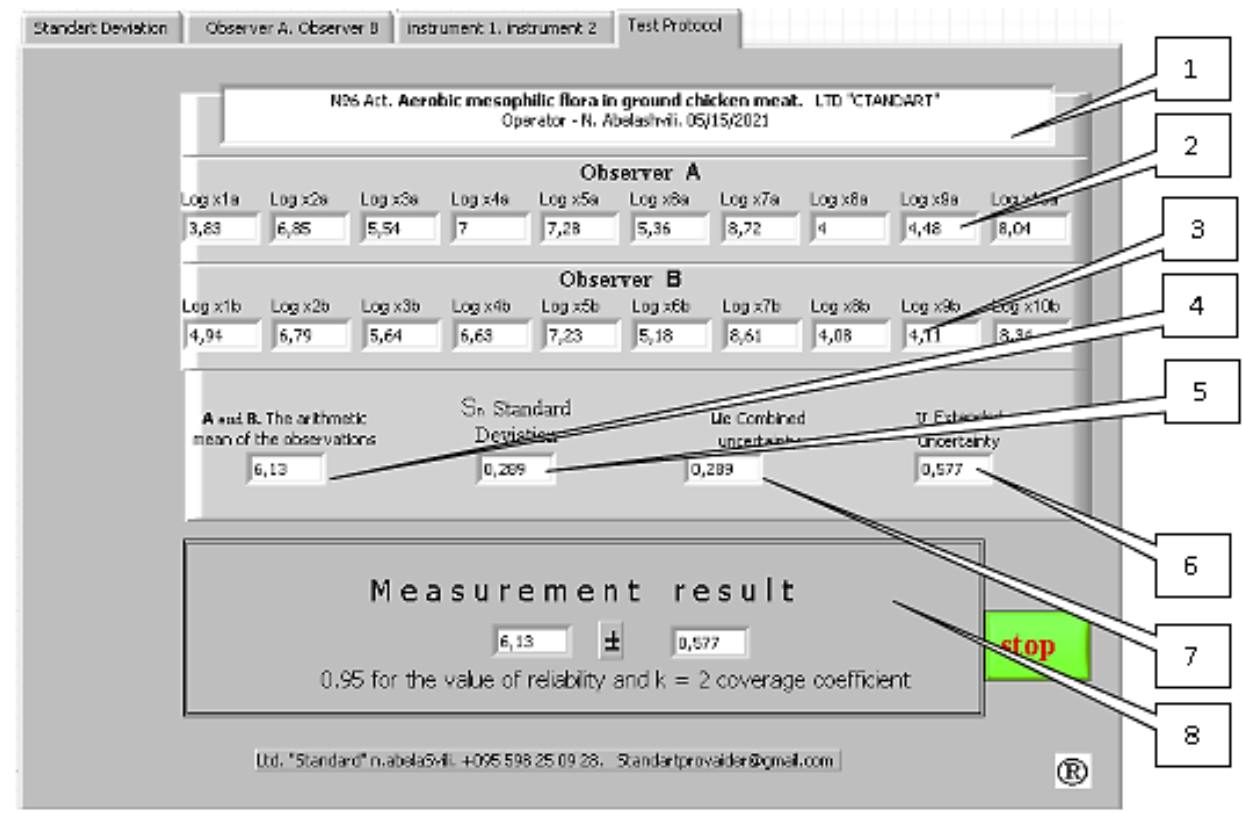

Рис. 4. Протокол испьтаний

Созданное программное обеспечение позволяет ускорит обработку экспериментальных данных, повысит точность и надежность микробиологического исследования, а также дает возможность применить данную разработку в качестве готового продукта для подставки в других измерительных и информационных системах как элемента встроенной системы.

Обсуждение результатов. Представленные окончательные результаты дают возможность однозначно судить о характере и свойствах как самого микробиологического эксперимента, так и оперативно оценить Точностные метрологические показатели, что особенно важно при проведении квалификационных испытаний.

На конкретном примере микробиологического анализа, рассмотренном в стандарте ISO/TS 19036:2019, подсчета аэробной мезофильной флоры в молотом курином мясе, показана эффективность разработанного программного обеспечения. В обеих расчетах получение денные совпали на $100 \%$.

Выводы. В статье рассматривается вопрос создания программного обеспечения для оценки количественного анализа микробиологического эксперимента с обязательным выделением доверительной вероятности, вида случайно распределения результатов измерений и оценки неопределённости микробиологического анализа пищевых продуктов и кормов для животных в среде LabVIEW и дается описание созданного продукта.

Применение среди графического программирования LabVIEW упрощает вопросы программирования, а также эксплуатационные показатели в силу легкости ввода данных и управления процессом вычислений.

Предложено и практически обосновано преимущество и адекватность применения данного программного средства.

\section{ЛИТЕРАТУРА}

1. GUM:1993, Guide to the expression of uncertainty in measurement, BIPM/IEC/IFCC/ISO/IUPAC/IUPAP/OIML;

2. ISO 19036:2019. Microbiology of the food chain - Estimation of measurement uncertainty for quantitative determinations;

3. ГОСТ Р 54502-2011 /ISO/TS 19036:2006. МИКРОБИОЛОГИЯ ПИЩЕВЫХ ПРОДУКТОВ И КОРМОВ ДЛЯ ЖИВОТНЫХ. Руководство по оценке неопределенности измерений при количественных определениях. Москва. Стандартинформ. 2012; [GOST R 54502-2011 / ISO / TS 19036: 2006. MICROBIOLOGY OF FOOD AND FEED FOR ANIMALS. Guidelines for Estimating Uncertainty of Measurement in Quantitative Determinations. Moscow. Standardinform. 2012]

4. EURACHEM/CITAC Guide. Quantifying Uncertainty in Analytical Measurement, 2nd edition, QUAM: 2000, available at http://www.eurachem.ul.pt/guides/quam.htm или http://www.measurementuncertainty.org/. 